\title{
Thoracolumbar and Lumbar Burst Fractures
}

\section{Sussan Salas, MD \\ Department of Neurological Surgery, Thomas Jefferson University Hospital, Philadelphia, PA}

Approximately 79,000 spinal fractures occur annually in the United States. Roughly three out of every four fractures involve either the thoracic or the lumbar spine ${ }^{1,2}$. The most common site of injury in the thoracic and lumbar spine is the thoracolumbar junction, the mechanical transition zone between the relatively rigid thoracic spine and the more flexible lumbar region ${ }^{3-5}$. The thoracolumbar junction receives 35 to $45 \%$ of the torsional and shear forces on the spine. This flexibility, combined with the curvature arising from biomechanical adaption to upright posture, makes the thoracolumbar

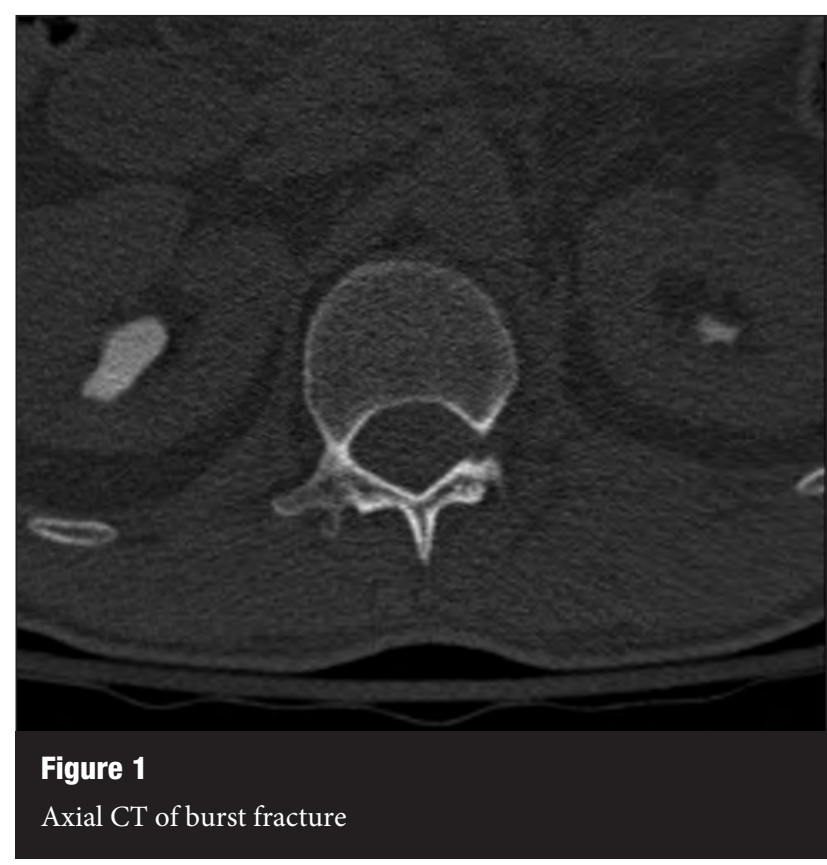

junction more prone to injury than the lumbar spine $^{6}$.

During standing, walking, or sitting upright, the vertebral column is under constant axial loading from gravity. A bending moment is also applied: the body's center of gravity is approximately $4 \mathrm{~cm}$ anterior to the first sacral vertebra, yielding a tendency to bend forwards (ventrally). This bending moment is countered by the posterior ligamentous complex (PLC). Through keeping the net sum of force vectors acting on the spine at zero, the spine's sagittal alignment remains stable. Trauma disrupting the PLC can impair the stability of the spine. Whiteside uses the analogy of a construction crane: when the cable holding the crane vertical breaks, the crane falls forward. Burst fractures and traumatic injuries to the thoracolumbar junction which compromise the PLC may produce this sort of "falling-forward" or kyphotic malalignment ${ }^{9}$.

Previous systems have attempted to predict the stability of traumatic injuries to the thoracic and lumbar spine and to anticipate instrumentation failures. Denis divides the spinal column into anterior, middle and posterior regions, classifying fractures by how the three regions are affected $^{3}$. In a burst fracture, the anterior and middle regions show compression failure, but the posterior region remains intact. McCormack has a 9-point grading scale (3 points on each of three characteristics) that determine the severity of load shifts from burst fractures; at 6 points or more, it is recommended that operative short segment posterior spinal instrumentation be augmented with anterior column support ${ }^{17}$. The Thoracolumbar Injury Classification and Severity Score (TLICS) system, designed by the Spine Trauma Study Group, scores patient fracture morphology, damage (if any) to the PLC, and neurological deficits seen. The TLICS score both estimates the relative need for surgical intervention and offers guidance as to operative approach if surgery is required ${ }^{13}$.
There remains considerable debate over when surgical intervention is necessary in thoracolumbar fractures. Goals of surgical intervention are to prevent neurological deterioration and to maximize functional and neurological recovery. Early and accurate neurological assessment is one key. Patients should be immobilized until fracture stability can be assessed adequately, to avoid new or worsening neurological deficits from spinal instability ${ }^{4}$.

The neurological exam is an important part of the initial trauma suvey, but optimally should be completed after hemodynamic stability has been established and patient vital signs stabilized. The exam should include tests of motor, sensory, sphincter, and reflex function ${ }^{10}$. While $70 \%$ of thoracolumbar injuries do not have associated neurological deficits ${ }^{2}$, these injuries potentially may involve either the spinal cord, the cauda equina, or individual lumbar nerve roots.

If the PLC is not damaged and there is no neurological deficit, a thoracolumbar junction burst fractures may be stable and the patient may be treated non-operatively with a brace. With neurological deficits, the recovery rate may be significantly higher with surgical intervention. The primary goal of operative therapy is decompression of the spinal canal, with coincident goal of stabilizing spinal elements to limit potential for instability-related further neurological decline $e^{4,7}$. Both anterior, posterior, and combined approaches are potentially available. Pattern of injury and location of neural element compression will guide choice of operative intervention. In appropriate patients, anterior decompression has a higher rate of neurologic improvement than posterior decompression ( $88 \%$ vs. $64 \%$ ) and a higher rate of recovery of bladder and bowl function $(69 \% \text { vs } 33 \%)^{8,18}$. Anterior decompression via corpectomy provides the maximal degree of canal decompression, but often must be augmented with posterior stabilization as well. Anatomic issues also must be considered; low lumbar corpectomies may be difficult due to psoas and vascular anatomy. Low lumbar (L3L5) burst fractures often require a posterior approach due to these concerns. 


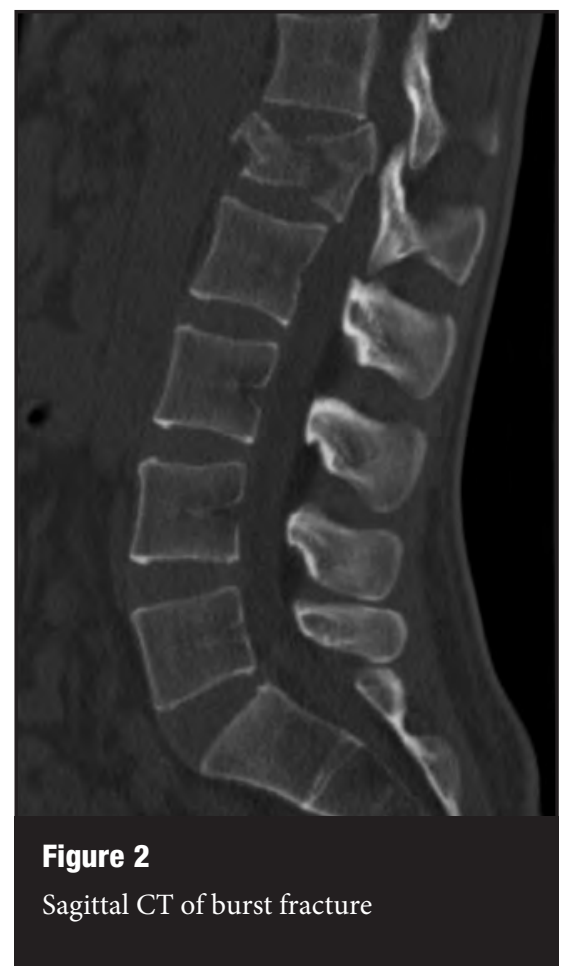

Thelong-termgoalofinstrumented stabilization is to maintain proper spinal alignment and stability until arthrodesis occurs ${ }^{9,19}$. Without solid fusion, metallic implants will eventually fail. Anterior stand-alone stabilization has the advantage of limiting loss of motion and restricting spinal arthrodesis to the levels immediately above and below injury. Anterior approaches require assistance of an approach surgeon, however, and carry the disadvantage possibile vascular, diaphragmatic, or enteric injury, and may be associated with higher risk of perioperative complications. Posterior short segment stabilization has a high rate of hardware failure, leading to longer constructs which may impair spinal mobility and predispose to future degenerative changes. When maintaining spinal flexibility is a priority, short-segment combined anterior and posterior stabilization may be considered ${ }^{17,19}$.

The role of surgical timing is unclear. Patients with progressive deficit need emergent decompression. Otherwise, most clinical studies have shown no association between timing and degree of neurologic recovery ${ }^{7,11}$.

\section{References}

1. Tran NT, Watson NA, Tender AF, et al. Mechanism of the burst fracture in the thoracolumbar spine. Spine 1995; 20:1984-8.

2. Hu R, Mustard CA, Burns C. Epidemiology of incident spinal fracture in a complete population. Spine 1996;21:492-9.

3. Denis F. The three column spine and its significance in the classification of acute thoracolumbar spinal injuries. Spine 1983;8:817-31.

4. Bohlman HH. Treatment of fractures and dislocations of the thoracic and lumbar spine. J Bone Joint Surg Am 1985;67:165-9.

5. Magerl F, Aebi M, Gertzbein SD, et al. A comprehensive classification of thoracic and lumbar injuries. Eur Spine J 1994;3:184-201.

6. Flanders AE. Thoracolumbar trauma imaging overview. Inst Course Lect 1999;48:429-31.

7. Benzel EC, Larson SJ. Functional recovery after decompressive operation for thoracic and lumbar spine fractures. Neurosurgery. 1986;19:772-8.

8. Bradford DS, McBride GG. Surgical management of thoracolumbar spine fractures with incomplete neurologic deficits. Clin Orthop. 1987;218:201-16.
9. Whitesides TE. Traumatic kyphosis of the thoracolumbar spine. Clin Orthop. 1977;128:78-92.

10. Holdsworth F. Fractures, dislocations, and fracture-dislocations of the spine. J Bone Joint Surg Am. 1970;52:1534-51.

11. Bradford DS, Akbarnia BA, Winter RB, et al. Surgical stabilization of fracture and fracture dislocations of the thoracic spine. Spine. 1977;2:185-96.

12. McCulloch PT, France J, Jones DL, et al. Helical computer tomography alone compared with plain radiographs with adjunct computed tomography to evaluate the cervical spine after high-energy trauma. J Bone Joint Surg Am. 2005;87:2388-94.

13. Rihn JA, Anderson DT, Vaccaro A, et al. A review of the TLICS system: a novel, user-friendly thoracolumbar trauma classification system. Acta Orthopaedica 2008; 79 (4): 461-6.

14. Keenen TL, Anthony J, Benson DR. Dural tears associated with lumbar burst fractures. J Orthop Trauma. 1990;4:243-5.

15. Holdsworth FW. Fractures, dislocations and fracture-dislocations of the spine. J Bone Joint Surg Br. 1963;45:6-20.

16. Mirza SK, Krengel WF, Chapman JR, et al. Early versus delayed surgery for acute cervical spinal cord injury. Clin Orthop. 1999;359:104-14.

17. McCormack T, Karaikovic E, Gaines R. The load sharing classification of spine fractures. Spine. 1994;19:1741-44.

18. Gertzbein SD. Scoliosis Research Society: multicenter spine fracture study. Spine. 1992;17:528-40.

19. McLain RF, Sparling E, Benson DR. Early failure of shortsegment pedicle instrumentation for thoracolumbar fractures: a preliminary report. J Bone Joint Surg Am. 1993;75:162-7.

\section{Brain power}

感 Jefferson.

Hospital for Neuroscience

1-800-JEFF-NOW

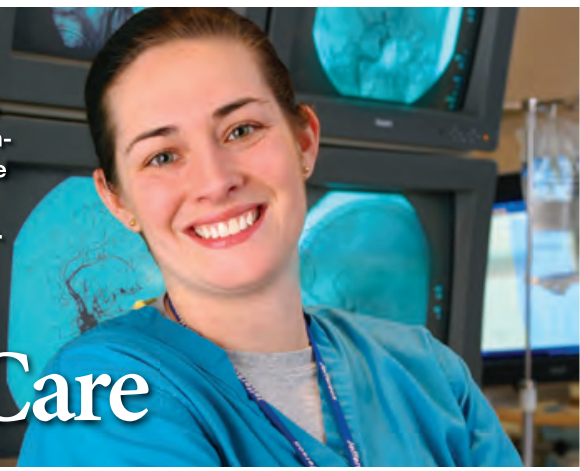

